\title{
A COVID-19 patient with intense burning pain
}

\author{
Feyzullah Aksan ${ }^{1}$ Eric Andrew Nelson ${ }^{2}$ (D) Kristin A. Swedish ${ }^{1}$
}

Received: 10 June 2020 / Revised: 17 July 2020 / Accepted: 22 July 2020 / Published online: 10 August 2020

(C) Journal of NeuroVirology, Inc. 2020

\begin{abstract}
A woman in her forties with asthma and COPD was admitted to a general medical floor with respiratory symptoms, body aches, and anosmia. Reverse transcription polymerase chain reaction detected severe acute respiratory syndrome coronavirus- 2 . Admission labs, including biomarkers of the systemic immunological dysfunction seen in many cases of coronavirus disease 2019 (COVID-19), were within normal ranges. On the second day of admission, she developed neck and back pain that was constant, burning in quality, and exacerbated by light touch and heat. Wearing clothing caused pain and interfered with her sleep. The area was tender to light finger stroke. The patient was given acetaminophen, NSAIDs, and opioids with no relief of pain. However, gabapentin was effective. At follow-up 1 month later, her symptoms were improved and still relieved by gabapentin. Neuropathic pain was seen in over 2\% of COVID-19 patients in one observational study. The pain seen in our case was bilateral, involved an area innervated by multiple levels of spinal nerves, and was limited to the back. While it is rare, a significant number of COVID-19 patients are afflicted by neuropathic pain, and our case illustrates that gabapentin may be effective.
\end{abstract}

Keywords COVID-19 $\cdot$ SARS-CoV-2 $\cdot$ Neuropathic pain $\cdot$ Neuralgia $\cdot$ Gabapentin

\section{Case description}

A woman in her forties came to a hospital with a 4-day history of worsening dry cough, dyspnea, generalized body aches, and chills. A day before the onset of these symptoms, she had lost her senses of taste and smell. A housemate had recently tested positive for severe acute respiratory syndrome coronavirus-2 (SARS-CoV-2) by reverse transcription polymerase chain reaction (RT-PCR). Our patient had hypertension, obesity, COPD, and asthma since childhood with one intubation more than 10 years earlier. She had smoked cigarettes on some but not all days since early adulthood. She took albuterol, budesonide/formoterol, montelukast, and losartan at home.

Except for elevated blood pressure and mild tachypnea, her vital signs were within normal limits. Her physical examination was unremarkable. RT-PCR detected SARS-CoV-2.

Eric Andrew Nelson

nelson7@gmail.com

1 Department of Internal Medicine, Montefiore Wakefield Campus, 600 East 233rd Street, Bronx, NY 10466, USA

2 Department of Psychiatry and Behavioral Sciences, Montefiore Medical Center, 111 E 210th Street, Bronx, NY 10467, USA
Admission labs, including biomarkers of immune dysfunction seen with coronavirus disease 2019 (COVID-19) (interleukin6, fibrinogen, D-dimer, lactate dehydrogenase, creatine kinase, and C-reactive protein), were within normal ranges. A chest $\mathrm{x}$-ray showed a small area of increased density overlying the lower left lung, and the patient was diagnosed with COVID-19 pneumonia. We continued her home medications and treated the patient with hydroxychloroquine and apixaban in accordance with our institution's protocol at the time. We also gave her oral prednisone and nebulized albuterol and ipratropium for presumed COPD and asthma exacerbation. She was not treated with a fluoroquinolone or any other antibiotic. On the second day of admission, she developed neck and back pain in the $\mathrm{C} 1$ to L4-L5 region, including the paraspinal area and trapezius muscle area. She reported the pain as constant, 7/10 in intensity, burning in quality, and exacerbated by light touch and warmth. Additionally, she said that wearing clothing caused pain and interfered with her sleep. There was no rash in the affected area. There was no history of similar episodes of pain or shingles. The area was tender to light finger stroke. She did not have photophobia, confusion, or focal neurologic deficits. We treated her with acetaminophen, an NSAID, oxycodone, and tramadol with no relief of pain. However, gabapentin relieved her pain, including the pain with wearing clothing and sensitivity to heat, allowing her to sleep comfortably. Her respiratory symptoms 
improved gradually during the 4-day hospitalization. At follow-up 1 month later, her pain had improved slightly and continued to be relieved by gabapentin.

\section{Discussion}

One of the most common symptoms of COVID-19 is pain. Muscle pain, joint pain, and headache are the types most frequently reported. Acetaminophen, NSAIDs, and narcotics are commonly prescribed; however, this patient's pain did not respond to those first-line treatments. The qualities of this patient's pain suggested a neuropathic etiology, so gabapentin was selected as a treatment. Gabapentin is effective against different types of neuropathic pain including thermal and mechanical allodynia (Bannister et al. 2017), and in addition to the qualities of the patient's pain, the efficacy of gabapentin further suggested a neuropathic etiology. The onset of the pain coincided with her SARS-CoV-2 infection, and there was no other likely explanation for the symptoms.

Human coronaviruses are known to be neuroinvasive, possibly using axonal transport for propagation, and have been associated with cases of encephalitis, acute flaccid paralysis, and Guillan-Barré syndrome (Desforges et al. 2019). Anosmia, likely due to viral invasion of the olfactory bulb, has been reported in a significant minority of COVID-19 patients (Tong et al. 2020). Interestingly, anosmia may signal a less severe course of disease; in one study, patients who presented with anosmia were ten times less likely to be hospitalized (Yan et al. 2020).

COVID-19 has spread worldwide, infecting millions and killing hundreds of thousands (https://coronavirus.jhu.edu/, accessed June 10, 2020). While neuropathic pain may be rarer than anosmia as a neurologic manifestation of COVID19 , it was found in 2.3\% ( $p=0.07)$ of hospitalized COVID-19 patients in one observational case series (Mao et al. 2020). However, no individual cases have been reported in detail, to our knowledge. There are several distinguishing features that differentiate the pain seen in our case from the most common viral neuropathic pain, herpes zoster. Herpes zoster, or shingles, is of course caused by reactivation of the varicella zoster virus years after an initial infection, while our case occurred soon after infection. Herpes zoster usually, but not always, manifests with a rash, and our patient had no cutaneous symptoms. Moreover, herpes zoster causes unilateral pain in a radicular or dermatomal pattern that often includes the anterior aspect of the level involved (Bennett and Watson 2009). The pain seen in our case was bilateral, involved an area innervated by multiple levels of spinal nerves, and was limited to the back. It is not a pattern previously described for a viral neuropathic pain, to our knowledge. Nerve injury due to pro-inflammatory cytokines and other mediators of the systemic immune dysregulation seen in some cases of COVID19 is unlikely as an explanation for this case given the normal levels of biomarkers.

\section{Conclusion}

COVID-19 can manifest with neuralgia, hyperalgesia, and allodynia. These symptoms were present in our patient alongside anosmia and without signs of systemic immune dysregulation or inflammation. While it is rare, a significant number of COVID-19 patients are afflicted by neuropathic pain, and our case illustrates that gabapentin may be effective in relieving it. Studies further exploring the etiology of neuropathic pain associated with COVID-19 would be beneficial.

Author contributions Dr. Feyzullah Aksan came up with the concept and wrote the initial story of the case. Dr. Aksan and Dr. Eric Nelson researched topics relevant to the case and wrote the discussion. Dr. Kristin Swedish gave suggestions and revised a later draft.

\section{References}

Bannister K, Qu C, Navratilova E, Oyarzo J, Xie JY, King T, Dickenson AH, Porreca F (2017) Multiple sites and actions of gabapentininduced relief of ongoing experimental neuropathic pain. Pain 158:2386-2395

Bennett GJ, Watson CP (2009) Herpes zoster and postherpetic neuralgia: past, present and future. Pain Res Manag 14:275-282

Desforges M, Le Coupanec A, Dubeau P, Bourgouin A, Lajoie L, Dubé M, Talbot PJ (2019) Human coronaviruses and other respiratory viruses: underestimated opportunistic pathogens of the central nervous system? Viruses 12:14. https://www.mdpi.com/about/ announcements/784

Johns Hopkins University School of Medicine (n.d.) Coronavirus Resource Center. https://coronavirus.jhu.edu/. Accessed on June 10th, 2020

Mao L, Jin H, Wang M, Hu Y, Chen S, He Q, Chang J, Hong C, Zhou Y, Wang D, Miao X, Li Y, Hu B (2020) Neurologic manifestations of hospitalized patients with coronavirus disease 2019 in Wuhan, China. JAMA Neurol 77:683-690

Tong JY, Wong A, Zhu D, Fastenberg JH, Tham T (2020) The prevalence of olfactory and gustatory dysfunction in COVID-19 patients: a systematic review and meta-analysis. Otolaryngol Head Neck Surg. https://doi.org/10.1177/0194599820926473

Yan CH, Faraji F, Prajapati DP, Ostrander BT, DeConde AS (2020) Selfreported olfactory loss associates with outpatient clinical course in Covid-19. Int Forum Allergy Rhinol 10:821-831

Publisher's note Springer Nature remains neutral with regard to jurisdictional claims in published maps and institutional affiliations. 